\title{
Distributed, Signal Strength based Indoor Localization Algorithm for Use in Healthcare Environments
}

\author{
Wyffels Jeroen, De Brabanter Jos, Crombez Pieter, Verhoeve Piet, Nauwelaers Bart, and De Strycker Lieven
}

\begin{abstract}
In current healthcare environments, a trend towards mobile and personalized interactions between people and nurse call systems is strongly noticeable. Therefore it should be possible to locate patients at all times and in all places throughout the care facility. The present article aims at describing a method by which a mobile node can locate itself indoors, based on signal strength measurements and a minimal amount of yes/no decisions. The algorithm has been developed specifically for use in a healthcare environment. With extensive testing and statistical support, we prove our algorithm can be used in a healthcare setting with an envisioned level of localization accuracy up to room revel (or region level in a corridor), while avoiding heavy investments since the hardware of an existing nurse call network can be reused. The approach opted for leads to very high scalability, since thousands of mobile nodes can locate themselves. Network timing issues and localization update delays are avoided, which ensures a patient can receive the needed care in a time and resources efficient way.
\end{abstract}

Index Terms-Received Signal Strength, Distributed Indoor Localization, Scalability, Low resources

\section{INTRODUCTION}

$\mathbf{S}$ YSTEMS capable in assisting medical staff efficiently, are highly valued in next generation nurse call systems. Current systems operate on a room oriented basis: when a patient launches a call for help, nurses are informed that in a certain room assistance is required. Suppose a patient is wearing a mobile alarm button by which he or she can make the request for assistance. Thus, nurses can be informed who needs the assistance, but since the patient is wearing a mobile node (i.e., a wristband), the patient could be anywhere in the care facility. If the call can be linked not only to the patients identity but also to the place of the call's origin, a wide range of possible applications and opportunities arise. Therefore, indoor localization of mobile nodes is needed. Existing indoor localization systems are all imparted by the necessary drawbacks, mostly to be defined in terms of installation costs, maintenance costs, scalability, robustness or localization accuracy. In the presented research, we combine the advantages and avoid the disadvantages of some of the

J. Wyffels is with the Department of Electrical Engineering ESAT, KU Leuven, Heverlee, Belgium, e-mail: jeroen.wyffels@kuleuven.be, and with Televic Healthcare, Izegem, Belgium

L. De Strycker, J. De Brabanter and B. Nauwelaers are with the Department of Electrical Engineering ESAT, KU Leuven, Heverlee, Belgium

P. Crombez is with Televic Healthcare, Izegem, Belgium

P. Verhoeve is with Televic Healthcare, Izegem, Belgium and is also a guest professor at Ghent University

Manuscript received August 15th, 2013; revised December 17th, 2013. existing systems. The proposed algorithm describes a method in which a mobile node can locate itself based on signal strengths between RF beacons. The envisioned localization accuracy is finetuned up to room level (or region level if the mobile node is inside a corridor). Since all mobile nodes are able to locate themselves, scalability issues are avoided. The research is supported by a statistical analysis (Support Vector Machines), and is presented as follows. Section II gives an outline of existing systems for indoor localization in general. In Section III, an overview of the developed indoor localization technique is given, followed by Section IV in which the performance of the proposed localization technique under laboratory conditions is given and analysed. Section V elaborates on the theoretical support for the developed indoor localization algorithm, after which a general conclusion about the performed work is presented in Section VI.

\section{RELATED WORK}

For wireless indoor localization purposes, several techniques can be applied which are typically based on Received Signal Strength (RSS), Time Of Arrival (TOA), on Angle Of Arrival (AOA), or on combinations of techniques which are well described by Goswami [1]. These techniques can be used with different technologies to obtain a location, i.e. Infrared technology, ultrasound, RF waves, magnetism, vision, etc. Each technology has its own advantages and drawbacks. Systems purely based on RF waves are widely used for indoor localization, since the localization accuracy can be high, depending on the used technique and available resources. RSS based systems are rather cheap systems since the majority of RF chips has an on board RSS block which determines the received power of the received signal. Mostly these RSS values are transformed into an estimated distance between the two transceivers communicating with each other. Different models for the relationship between distance and received power exist, but all conversion algorithms are subject to indoor propagation effects (fading, scattering, absorption, reflection etc.), making the relationship between distance and received power both dubious and unreliable. The same remark applies for numerous systems which try to perform indoor localization with a limited amount of RF beacons, whether they are WIFI based or not. An extensive overview of indoor propagation effects is given in the book by Barclay [2]. Avoiding indoor propagation effects for indoor localization purposes can be done by making use of radio maps/fingerprinting techniques. The localization 
accuracy of fingerprinting based systems is subject to changes in the environment where the localization procedure takes place. For healthcare situations, with a lot of moving objects such as beds, standard fingerprinting techniques are thus unusable. Techniques for adapting the radio map to environmental changes as proposed by Yin et al. [3] boast a limited number of RF beacons. These discussed techniques yield problems in determining if a mobile node is just inside or just outside a room, which is the main topic of research in our work. Altintas et al. describe a method for RSS based indoor localization, using a short term memory to filter out impossible localization outcomes due to the limited moving speed of a person [4]. The proposed technique, however, is based on WIFI which uses a lot of energy, and the achieved localization accuracy $( \pm 2 \mathrm{~m})$ is unacceptable since it cannot be guaranteed that a mobile node inside a room won't get localized in a corridor instead of inside the room. Several systems which implement indoor localization in the application field of the healthcare sector instead of generically applicable systems, exist too. Shirehjini et al. presented a system using RFID (Radio Frequency IDentification) for medical equipment localization in healthcare facilities [5]. Although the proposed system performs well in terms of indoor localization accuracy using passive RFID tags, the system is susceptible to misalignment of the tags compared to the RFID readers which is exactly what cannot be guaranteed when persons are wearing these RFID tags. The nature of an RFID system implies high installation costs, since RFID readers as well as network connections to these readers must be foreseen (the passive tags themselves are rather cheap). All RFID readers should be able to send the ID of the found tags in range to a central server, possibly causing network timing and/or scalability issues. Ultrasound based systems capable of locating people or assets in a healthcare facility have also been the main topic of research, as well as systems using WIFI networks. However, the installation of such a system requires the deployment of a stand alone network besides the existing nurse call networks, making it an expensive solution. Moreover, these systems have problems in determining whether a mobile node should be located just within or just outside a patient room, resulting in a too low localization accuracy which cannot be tolerated in possibly life threatening situations. Chen et al. described a patient monitoring method using ZigBee, in which the ZigBee mesh network is used for sending vital signs to nurses as well as for the indoor localization part [6]. Such and similar systems typically use broadcast or multicast schemes to ensure the delivery of the location updates and/or the vital signs of a patient, but the high network traffic caused by this approach induces scalability and timing issues. More commercial systems are discussed in the work of $\mathrm{Gu}$ et al. [7]. They concluded that no currently available localisation system is capable of ensuring scalability and availability of location information at all times at all places, and therefore using several techniques or systems together may lead to an indoor localization systems which works in all cases. The need of a system which is optimized for this typical field of application and copes with all aforementioned challenges is clear.

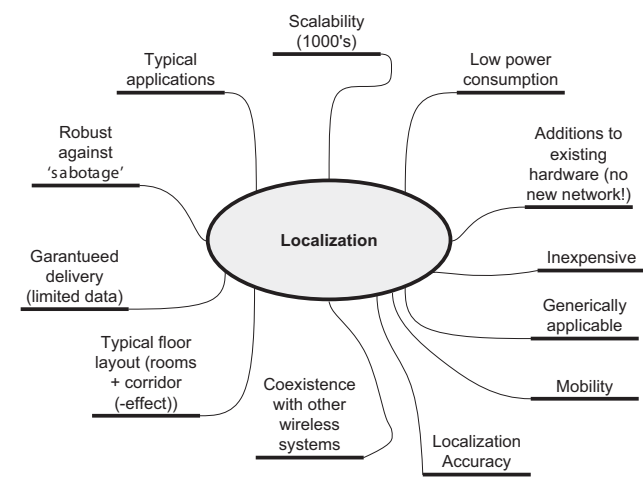

Fig. 1. Indoor localization constraints and prerequisites for healthcare facilities.

\section{PROPOSED INDOOR LOCALIZATION TECHNIQUE}

\section{A. Indoor localization constraints and prerequisites}

Indoor localization in healthcare facilities is subject to several technological constraints and prerequisites which are visualized in Fig. 1. Possibly thousands of mobile nodes would need to be located within a certain time frame. According to Ottoy et al., this can result in scalability issues when trying to perform localization of mobile nodes on a centralized server [8]. The approach proposed in the present paper excludes the aforementioned setbacks by focusing on indoor localization on the mobile node itself. The node communicates its position to the backbone only when required, implying a good system scalability. Existing nurse call systems have multiple access points in each room, which makes it possible to have a lot of wireless connection possibilities to the nurse call system backbone. To each of these existing access points, a RF chip can be attached (in this research, the RF chip is a Bluetooth Low Energy (BLE) Texas Instruments CC2540 SOC [9]). Since these chips have a built in RSS block, it is possible to get a quick indication of the position of a mobile node relative to a nurse call system access point. By using a high number of beacons per unit of surface on top of the existing nurse call network, minimal infrastructure costs are needed, while accurate indoor localization can be obtained if an appropriate algorithm is used. From a practical point of view, the target localization accuracy is up to room level for a nurse call originating in a room. If the call originated in a corridor, any localization attempt should result in the correct region within that corridor from where the call originated. To conclude, a $100 \%$ guarantee must be obtained that the withdrawn localization result actually complies with the real location of the mobile node, even if a lot of movements around the mobile node are happening, if the mobile node gets covered (system robustness), or if other wireless systems are installed on site.

\section{B. Envisioned method of localization}

Suppose all existing access points to the nurse call network are equipped with a BLE chip. All RF beacons send advertising packets on the dedicated advertising channels (channel 37 , 38 or 39) [10]. These packets are broadcasted within a certain period $\mathrm{T}$ _advEvent $=$ advInterval + advDelay. In present 


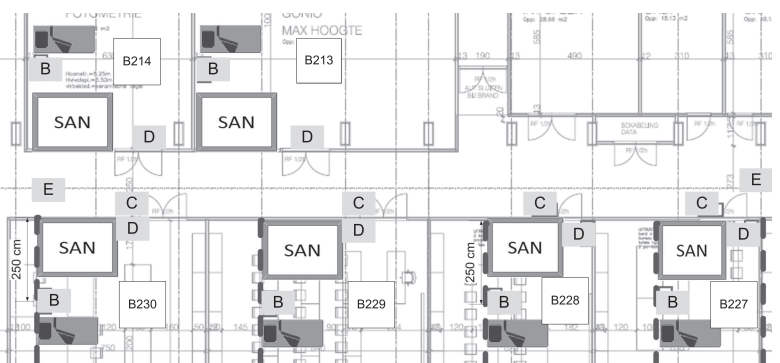

Fig. 2. Test set-up

research, an advInterval of $20 \mathrm{~ms}$ is used, thus maximizing the advertising frequency. AdvDelay is a pseudo random delay between $0 \mathrm{~ms}$ and $10 \mathrm{~ms}$, thus on average 40 advertising packets per second can be expected from each continuously broadcasting beacon. On reception of an advertising packet, the receiver can determine the MAC address of the sending beacon as well as the power by which the advertising packet has been received and store this information in a local database. A CC2540 has a primary MAC address assigned by the manufacturer, and a secondary, user assignable MAC address which is used in present research. Location information can be embedded in this secondary MAC address [11]. The first three bytes of the secondary MAC address are reserved for future use, while the fourth byte indicates the physical location of the beacon related to a room. This fourth byte indicates near which type of nurse call access point the BLE chip has been installed. In our system, five types of RF beacons are used: 1) 'AA': Inside a patient room near the sanitary facilities, 2) ' $\mathrm{BB}$ ': inside a patient room near the bed, 3) 'CC': in a corridor, above an entrance door to a patient room, 4) 'DD': in a patient room, near the entrance door, and 5) 'EE', at the end of a corridor. These five types are denoted respectively as A, B, C, $\mathrm{D}$ and $\mathrm{E}$ in Fig. 2. The fifth and sixth byte determine the room itself: the building name, the floor level and the room number on that specific floor are indicated by these numbers. For instance, when a mobile node receives an advertising packet from CC:EE:FF:CC:G3:78, this means the beacon is installed inside the corridor (' $\mathrm{CC}$ ') in building ' $\mathrm{G}$ ', floor 3 (' $\mathrm{G} 3$ '), outside room 78 ('78'). Thus, a mobile node can quickly gain knowledge about its position relative to all beacons in range, by just analysing their advertising packets. Since there is no need for a connection to be established between beacons and mobile nodes, nor a database to be accessed to link a MAC address to a location, using this approach avoids scalability issues. To estimate its position from the captured information, additional processing on the mobile node is needed which consequently shouldn't require a lot of computing resources. This procedure is explained in Paragraph III-C.

\section{Implemented algorithm}

1) Overall localization cycle: For the experiments, some laboratories and a corridor of KU Leuven's campus Ghent have been used, since they compare to a healthcare facility. In Fig. 2, a graphical overview of the test set-up is given. A general overview of the localization process is given in Fig. 3. Whenever the localization cycle is initiated, the mobile node

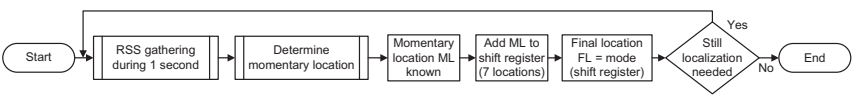

Fig. 3. Overall localization procedure

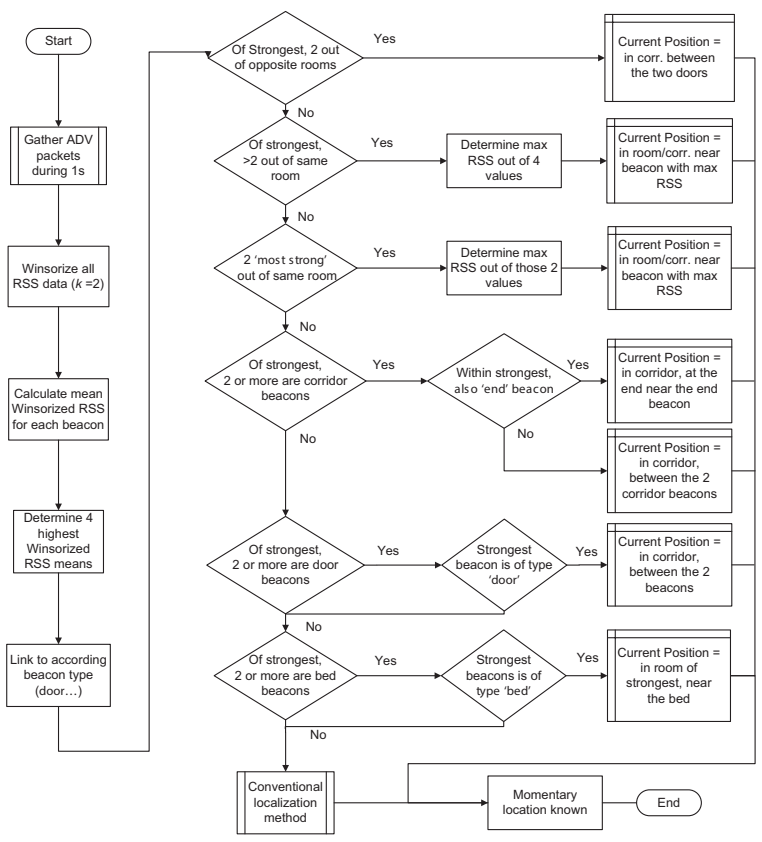

Fig. 4. Momentary localization procedure

starts with gathering advertising packets during one second and put them in a local database. After this, the momentary location gets determined as described in Paragraph III-C2. Out of the momentary localization procedure, a location is withheld, which is added to a 'shift register of locations' of size seven. The most occurring location in this shift register is the final localization process outcome, which can be reported to the nurse call system backbone. One can get an indication of the stationariness of the mobile node. If the shift register is filled with the same locations, meaning seven consecutive momentary localization procedures had the same outcome, we can assume the mobile node is stationary. If the most occurring element in the shift register only occurs two or three times in the shift register, we can expect 1) the mobile node itself moves around, or 2) environmental changes around the mobile node (people passing). The localization outcome may thus temporarily change. However, since these are only temporary effects, the most occurring element in the shift register will remain the same and thus the overall localization procedure will not return a false location.

2) Momentary localization procedure: A decision tree as can be found in Fig. 4 has been developed for the actual localization procedure, and can be seen as a sophisticated proximity algorithm. All captured advertising data is stored and linked to the respective beacons of origin. After this, a Winsorized mean is calculated for all RSS values linked to the same beacon. The Winsorized mean is a robust estimator of the mean RSS value per beacon, which is relatively insensitive to outliers caused by moving people, indoor propagation effects etc. The $k$-times Winsorized mean can be calculated as 
$\bar{X}_{w k}=\frac{1}{n}\left((k+1) X_{k+1}+\sum_{i=k+2}^{n-k-1} X_{(i)}+(k+1) X_{(n-k)}\right)$ where $n$ is the number of RSS values which can be related to the same beacon. $X_{(i)}$ is the $i$-th order statistic if the measurements are rearranged in an ascending order: $X_{(1)} \leq X_{(2)} \leq$ $\ldots \leq X_{(n)}$ [12]. In this research, the Winsorization procedure incorporates the calculation of the $\left(k=\frac{n}{5}\right)$-times Winsorized mean. The four highest mean RSS values are used for further processing. These obtained values can be automatically related to the four respective beacons, and knowledge about the four accompanying beacon types is gained. We sort these beacons from the beacon with the highest to the beacon with the fourth highest mean Winsorized RSS value. Six possible cases are then analysed. If the two strongest received beacons out of the four beacons can be related to two rooms situated opposite to each other (corridor in between), the withdrawn location of the mobile node is in the corridor between the two entrance doors of the respective rooms. Should this not be the case, it is possible out of the four beacons, more than two (including or not including thé strongest) can be related to the same room. In that case, the algorithm concludes the position of the mobile node is inside or outside this room, but in all cases near the beacon with the highest mean Winsorized RSS value. Whenever the previous cases didn't apply for the gathered set of RSS data, the algorithm checks if the two highest mean Winsorized RSS values are related to one room. If the two strongest mean Winsorized RSS values can be linked to the same room, the algorithm concludes that the location of the mobile node is inside or outside this room, taking into account that the final inside/outside decision is made by the type of the strongest received beacon. This brings us with the fourth possible case, when two or more of the four highest Winsorized means can be related to two or more corridor beacons. A distinction has to be made here: in a corridor, two types of beacons are present (' $\mathrm{C}$ ' and ' $\mathrm{E}$ ' type). In case one of the four strongest mean Winsorized RSS values can be related to an end node, the algorithm decides the mobile node is located at the end of a corridor. Should this not be the case, the concluded location is inside the corridor, between the two strongest received corridor nodes. If also this fourth case is not valid, still two more options are included in our decision tree. If of the four strongest, two or more are door nodes and the strongest is also of the door beacon type, the algorithm decides the mobile node can be located between the two doors which have the strongest mean Winsorized RSS. This can be the case when the mobile node is in between the doors of two oppositely situated rooms inside a corridor. As a final typical case, two or more of four strongest Winsorized mean RSS values could be linked to bed type beacons. In healthcare environments, it is typical that a wall is dividing two rooms, with two beds symmetrically placed around this wall. In this case, possibly the mentioned two bed beacons can be received very strong (the two strongest), however a wall is dividing both rooms. In this case, the algorithm should decide the mobile node is located near the bed (and thus in the room) of the strongest received bed beacon. If none of the aforementioned possibilities occur, we have to fall back on the estimation of a distance between sender and receiver, which is what we want to avoid in the proposed algorithm. We optimized our decision tree as such it minimizes the number of times this distance estimation has to be done, but in some cases, there's no other way. The mobile node could then locate itself by some triangulation algorithm which takes a lot of time, or hand all available data during the one second interval to the central backbone. The latter might induce scalability issues on the central localization server, when too much mobile nodes hand their data to this server at the same time. In the following section, an overview on the obtained localization results is presented.

\section{RESULTS}

In Table I, an overview is given of the performance of the decision tree. RSS measurements were gathered during 150 seconds at different sample points. In a room, measurements were gathered once at a distance of one metre from a ' $\mathrm{B}$ ' beacon, and once at a distance of one metre from a ' $D$ ' beacon. In a corridor, measurements were taken at one metre from each ' $C$ ' beacon and from each ' $E$ ' beacon. Also in the middle between two consecutive ' $C$ ' beacons, measurements were gathered. This resulted in 23 sample points. For each of the gather points and for each of the 150 sample seconds, the localization procedure was initiated, resulting in a determined location which was compared with the actual one. The localization accuracy over these 150 seconds is averaged over all similar locations (in a room/in a corridor). The envisioned localization accuracy is denoted with RLA (Room Level Accuracy (inside room)/Region Level Accuracy (in corridor)). The test has been conducted in two separate environments: a full drywall environment and a full brick environment. A full brick wall is supposed to influence RF signals to a higher extent compared to a drywall (thus, drywall environments are worst case scenarios). Also, the beacon level accuracy (BLA) is presented. An investigation is performed in how much of the localization procedures the decision tree is able to determine which is the nearest beacon, more than only decide in which room the mobile node can be situated. Localization with beacon level accuracy is not the envisioned topic of research, but it can give an indication about the possibilities of using a decision tree as proposed in this research, when beacon level localization accuracy is needed. Out of Table I, it can be seen the localization of a mobile node is possible on a distributed scale based on the proposed decision tree. However, the influence of the size of the shift register of locations (FilterWindowSize FWS) which is discussed in Section III-C becomes clear. When the shift register functionality isn't used (FWS $=1$ ), we see the RLA performance is falling slightly compared to a FWS starting from 3 on (odd numbers only). The larger the FWS, the better the RLA becomes. In the envisioned field of application however, it may not take too long for an accurate location update to be reported to the nurse call system. The larger the FWS size, the slower the update rate of the localization outcome, since the most occurring element in the shift register is withdrawn as the location to report to the backbone. An update delay of maximum five seconds is acceptable from a 
user point of view, meaning the maximum allowable FWS is a shift register of nine locations. After five seconds, the most occurring element will then be the new location. The gain in RLA when FWS $>9$ is marginal, indicating a location update delay of maximum five seconds is achievable. In neither of the two environments, a $100 \%$ BLA can be obtained using the decision tree of Fig. 4 but the RLA reaches $100 \%$ in both environments.

TABLE I

DECISION TREE PERFORMANCE

\begin{tabular}{c|cc|cc}
\hline & \multicolumn{2}{|c|}{ Drywall } & \multicolumn{2}{c}{ Full Brick } \\
\hline FWS & RLA [\%] & BLA [\%] & RLA [\%] & BLA [\%] \\
\hline 1 & 99,2 & 87,4 & 99,8 & 99,9 \\
3 & 100,0 & 97,5 & 100,0 & 92,3 \\
5 & 100,0 & 97,8 & 100,0 & 91,8 \\
7 & 100,0 & 97,9 & 100,0 & 91,5 \\
9 & 100,0 & 98,0 & 100,0 & 92,0 \\
11 & 100,0 & 98,1 & 100,0 & 91,8 \\
13 & 100,0 & 98,3 & 100,0 & 92,2 \\
15 & 100,0 & 98,3 & 100,0 & 92,4 \\
\hline
\end{tabular}

\section{DisCUSSION}

\section{A. Theoretical support}

The adoption of the decision tree, being a sophisticated proximity localization technique, was motivated by the fact that RSS based multi-lateration gives bad performance for indoor localization. Sophisticated statistical algorithms, although having the potential for excellent good localization, are too computationally intensive and too slow for a realtime implementation on a mobile node with limited power and computational resources. The proposed decision tree structure is designed based on general knowledge of wave propagation. In the proposed decision tree, several parameters have been used, namely 1) Size of the shift register, 2) Number of RSS values to gather before the momentary localization cycle can be initiated, 3) Using only the four strongest mean Winsorized RSS values, and 4) Number of distinct yes/no decisions before initiating a conventional localization algorithm. Although the used parameter settings lead to acceptable localization results with the given prerequisites and constraints out of Fig. 1, the question remains if these parameter settings are the best option. A discussion on the size of the shift register has already been given. On the one hand it might be possible to further optimize the decision tree structure and parameters. On the other, however, inherent physical limitations which are not a priori known have influence on the attainable accuracy. Statistical algorithms can help here in two ways. Suppose the localization result of a sophisticated statistical algorithm is the best achievable result. A comparison between this golden standard' and the localization result of the decision tree can be made. This could give a general idea of how much room for improvement is still left. Secondly, statistical algorithms can give information on the optimal value of some parameters. In the decision tree, for example, the four strongest mean Winsorized RSS values are used. This cannot be changed easily in the decision tree as its whole structure is based on it. The estimation of the location as it is done by the decision tree can be seen as classification algorithm: given a set of input data (here: vector containing mean Winsorized RSS values), what should be the output class (here: one of the possible locations)? Classification problems may be solved by statistical means such as Support Vector Machines (SVM) applicable to a broad category of classification problems [13]. Support Vector Machines can cope with binary classification problems (only two output classes possible), even though multi-class classification problems can be solved too as explained by Suykens et al. [14]. In this research, Least Squares-Support Vector Machines are used.

1) Method: LS-SVM (Least Squares-Support Vector Machines) [14] In case of a binary classification problem, we can explain the LS-SVM classification problem as follows. Given a training set defined as $\mathcal{D}_{n}=\left\{\left(X_{k}, Y_{k}\right): X_{k} \in \mathbb{R}^{d}, Y_{k} \in\right.$ $\{-1,+1\} ; k=1, \ldots, n\}$, where $X_{k}$ is the $k$-th input pattern and $Y_{k}$ is the $k$-th output pattern. In the primal weight space, LS-SVM for classification is formulated as

$$
\begin{aligned}
\min _{w, b, e} & \mathcal{J}_{c}(w, e)=\frac{1}{2} w^{T} w+\frac{\gamma}{2} \sum_{i=1}^{n} e_{i}^{2} \\
\text { s.t. } \quad Y_{i}\left[w^{T} \phi\left(X_{i}\right)+b\right] & =1-e_{i}, \quad i=1, \ldots, n
\end{aligned}
$$

where $\phi: \mathbb{R}^{d} \rightarrow \mathbb{R}^{n_{h}}$ is the feature map to the high dimensional feature space (can be infinite dimensional) as in the standard Support Vector Machine (SVM) case [15], $w \in \mathbb{R}^{n_{h}}, b \in \mathbb{R}$ and $\gamma \in \mathbb{R}_{0}^{+}$is the regularization parameter. On the target value an error variable $e_{i}$ is allowed such that misclassifications can be tolerated in case of overlapping distributions. By using Lagrange multipliers, the solution of (1) can be obtained by taking the Karush-Kuhn-Tucker (KKT) conditions for optimality [16]. The result is given by the following linear system in the dual variables $\mu$

$$
\left[\begin{array}{c|c}
0 & Y^{T} \\
\hline Y & \Omega^{(c)}+\frac{1}{\gamma} I_{n}
\end{array}\right]\left[\begin{array}{c}
b \\
\hline \mu
\end{array}\right]=\left[\begin{array}{c}
0 \\
\hline 1_{n}
\end{array}\right],
$$

with $Y=\left(Y_{1}, \ldots, Y_{n}\right)^{T}, \quad 1_{n}=(1, \ldots, 1)^{T}$, $\mu=\left(\mu_{1}, \ldots, \mu_{n}\right)^{T}$ and $\Omega_{i l}^{(c)}=Y_{i} Y_{l} \phi\left(X_{i}\right)^{T} \phi\left(X_{l}\right)=$ $Y_{i} Y_{l} K\left(X_{i}, X_{l}\right)$ for $i, l=1, \ldots, n$ with $K(\cdot, \cdot)$ a positive definite kernel. Such a positive definite kernel $K$ guarantees the existence of the feature map $\phi$ but $\phi$ is often not explicitly known. Based on Mercer's theorem, the resulting LS-SVM model for classification in the dual space becomes

$$
\hat{y}(x)=\operatorname{sign}\left[\sum_{i=1}^{n} \hat{\mu}_{i} Y_{i} K\left(x, X_{i}\right)+\hat{b}\right]
$$

where $K: \mathbb{R}^{d} \times \mathbb{R}^{d} \rightarrow \mathbb{R}$. For example, the Gaussian kernel $K\left(X_{i}, X_{j}\right)=(1 / \sqrt{2 \pi}) \exp \left(-\left\|X_{i}-X_{j}\right\|_{2}^{2} / 2 h^{2}\right)$ with bandwidth $h>0$.

2) Simulation results: The LS-SVM toolbox [17] is uses to support the parameter choices in the decision tree. All mean Winsorized data gathered at different gathering points (which comply with the position of the beacons mentioned in Fig. 2) are put together in one large dataset containing 3450 different sample points, resulting in a dataset of size $3450 \times 23$, with 23 the number of possible RF beacons. For each of these 3450 rows, we label each one of them: these labels comply with 
TABLE II

LS-SVM LOCALIZATION POSSIBILITIES

\begin{tabular}{c|ccccc}
\hline \multirow{2}{*}{ Number of output classes } & \multicolumn{5}{|c}{ Localization accuracy if input data $=\ldots$} \\
2 strongest & 3 strongest & 4 strongest & 5 strongest & All data \\
\hline 13 & $98,87 \% \pm 0,27 \%$ & $99,15 \% \pm 0,28 \%$ & $99,19 \% \pm 0,23 \%$ & $99,24 \% \pm 0,25 \%$ & $99,35 \% \pm 0,22 \%$ \\
17 & $0,02 \% \pm 0,05 \%$ & $0,04 \% \pm 0,05 \%$ & $99,04 \% \pm 0,25 \%$ & $99,15 \% \pm 0,26 \%$ & $99,25 \% \pm 0,24 \%$ \\
23 & $0,01 \% \pm 0,03 \%$ & $0,01 \% \pm 0,03 \%$ & $98,94 \% \pm 0,34 \%$ & $99,04 \% \pm 0,31 \%$ & $99,19 \% \pm 0,24 \%$ \\
\hline
\end{tabular}

one of the 23 actual possible locations and thus a matrix of $3450 \times 1$ labels is created. The dataset and the accompanying labels are then split into two parts $(2300 \times 23+1150 \times 23$ and $2300 \times 1+1150 \times 1$ parts, respectively). We call the $2300 \times 23$ dataset the training dataset, and the $1150 \times 23$ dataset the test dataset. The toolbox builds a Least Squares-Support Vector Machine on the training dataset and determines suitable tuning parameters. After the model has been built, the performance of the Support Vector Machine can be determined: we let the LSSVM toolbox now use the test dataset and the created model classify the data, checking afterwards if the assigned label does or doesn't comply with the manually assigned label. If the toolbox is capable of assigning the label correctly, we assume the classification process in practice can be done, based on the training dataset. However, if the toolbox isn't capable of classifying the test dataset, this means there wasn't enough input data in the training dataset to build a suitable model. By lowering the amount of input data in the training set, an analysis is conducted on how much input data is really needed to base a model on. Several test cases were investigated, of which the results can be found in Table II. All of the tests have been repeated 100 times for statistical relevance with shuffled training and test datasets. For each of the 23 investigated locations, 3 different number of output classes were researched:

1) 13 output classes. In this case, too much location abstraction is made. No distinction between inside or outside a room (thus in corridor) can be made any more.

2) 17 output classes. This case complies with localization up to the envisioned level of accuracy: a distinction between in- and outside a room can be determined, as well as the correct region within a corridor.

3) 23 output classes. This complies with localization up to beacon level (which is not the envisioned level of localization accuracy in this research).

For each iteration, the mean multi-class classification performance was calculated for each of the 23 locations. The mean LS-SVM performance over the 100 iteration means ( \pm standard deviation) related to the same actual location and number of output classes is also determined, and presented in Table II. The influence of the amount of input data becomes clear. If data from all beacons in range is used, the toolbox is able to determine the location of all analysed number of output classes in almost every case. If we lower the amount of input data and only use a number of highest mean winsorized RSS values, we see the performance of the toolbox decreases slightly when less data is used when building the model. When too few data is available (in our case, only the 3 highest mean Winsorized
RSS values), the toolbox fails to classify the test set: only when too few output classes (13) are used, the toolbox can still classify correctly. Since a statistical toolbox is unable to classify incoming data when less than the four strongest mean Winsorized RSS values are used and 17 output classes are needed, using the four strongest received beacons in our proposed algorithm is advisable.

\section{CONCLUSIONS}

A decision tree for distributed indoor localization based on RSS for use in healthcare environments is presented. By exploiting the typical healthcare facility building topology, indoor localization proved to be feasible on a distributed scale given that a high number of beacons per unit of surface are in place. In the proposed system, false localization results, scalability issues and heavyweight algorithms are avoided, while the existing nurse call network installations can be reused to reduce the installation and maintenance costs. The proposed distributed indoor localization algorithm is based on simple yes/no decisions to reduce the system complexity and location calculation time. All fixed BLE beacons broadcast continuously location information. This enables the mobile nodes to quickly get an indication about their location. By applying the proposed decision tree, the mobile nodes can accurately locate themselves, thus avoiding network scalability and timing issues. The envisioned level of localization accuracy can be met when using this kind of algorithms on the mobile node itself, when enough RF beacons are installed in the care facility. A theoretical approach supporting our findings has also been presented. Performing indoor localization based on RSS on a distributed scale has proven to be feasible. A low cost and low resources localization system can work accurately on a distributed and resources efficient base, whether people or expensive assets such as heart rate monitors must be tracked.

\section{ACKNOWLEDGEMENT}

This Ph.D. research is funded by IWT, the Flemish agency for Innovation by Science and Technology (Baekeland mandate), and is a cooperation between KU Leuven (DraMCo Research Group) and Televic NV.

\section{REFERENCES}

[1] S. Goswami, Indoor Location Technologies. Springer New York, 2013, pp. 83-103.

[2] L. W. Barclay, Propagation of Radiowaves, 2nd ed., L. Barclay, Ed. The Institution of Engineering and Technology, 2002.

[3] J. Yin, Q. Yang, and L. Ni, "Learning Adaptive Temporal Radio Maps for Signal-Strength-Based Location Estimation," Mobile Computing, IEEE Transactions on, vol. 7, no. 7, pp. 869-883, 2008. 
[4] B. Altintas and T. Serif, "Indoor Location Detection with a RSS-based Short Term Memory Technique (KNN-STM)," in Pervasive Computing and Communications Workshops (PERCOM Workshops), 2012 IEEE International Conference on, 2012, pp. 794-798.

[5] A. Shirehjini, A. Yassine, and S. Shirmohammadi, "Equipment Location in Hospitals Using RFID-Based Positioning System," IEEE Transactions on Information Technology in Biomedicine, vol. 16, no. 6, pp. 1058-1069, Nov 2012. [Online]. Available: http://ieeexplore.ieee.org/lpdocs/epic03/wrapper.htm?arnumber=6328280

[6] S.-K. Chen, T. Kao, C.-T. Chan, C.-N. Huang, C.-Y. Chiang, C.-Y. Lai, T.-H. Tung, and P.-C. Wang, "A Reliable Transmission Protocol for ZigBee-Based Wireless Patient Monitoring," IEEE Transactions on Information Technology in Biomedicine, vol. 16, no. 1, pp. 6-16, Jan 2012. [Online]. Available: http://ieeexplore.ieee.org/lpdocs/epic03/wrapper.htm?arnumber=6044716

[7] Y. Gu, A. Lo, and I. Niemegeers, "A survey of indoor positioning systems for wireless personal networks," Communications Surveys Tutorials, IEEE, vol. 11, no. 1, pp. 13-32, 2009.

[8] G. Ottoy, A. Van Nieuwenhuyse, K. D'hoe, J.-P. Goemaere, and L. De Strycker, "Improving the performance of a RSS-based Location Estimation System, Study and Evaluation," in Proceedings of the International Conference on Positioning and Context-Awareness, Artesis University College. 25-29, 2009, ISBN 9789080758704.

[9] Texas Instruments, CC2540 datasheet, 10 2010. [Online]. Available: http://www.ti.com/lit/ds/symlink/cc2540.pdf

[10] Bluetooth V4.0 and Bluetooth Low Energy. https://www.bluetooth.org/Technical/Specifications/, Bluetooth SIG Std.

[11] J. Wyffels, J. P. Goemaere, P. Verhoeve, P. Crombez, B. Nauwelaers, and L. De Strycker, "A Novel Indoor Localization System for Healthcare Environments," in 25th International Symposium on Computer-Based Medical Systems (CBMS), 2012. IEEE, Jun 2012, pp. 1-6.

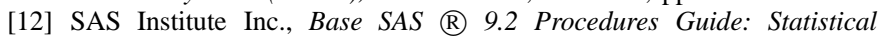
Procedures, 3rd ed. SAS Institute Inc., Cary, NC, USA, 2010.

[13] S. Abe, Support Vector Machines for Pattern Classification, 2nd ed. Springer-Verlag, 2010

[14] J. A. K. Suykens, T. Van Gestel, J. De Brabanter, B. De Moor, and J. Vandewalle, Least Squares Support Vector Machines. World Scientific, 2002

[15] V. N. Vapnik, The Nature of Statistical Learning Theory. SpringerVerlag, 1999.

[16] D. P. Bertsekas, Nonlinear Programming, 2nd ed., 1999.

[17] K. De Brabanter, P. Karsmakers, F. Ojeda, C. Alzate, J. De Brabanter, K. Pelckmans, B. De Moor, J. Vandewalle, and J. A. K. Suykens, "LSSVMlab Toolbox User's Guide - Version 1.8," KU Leuven, Tech. Rep. 10-146, 2010.

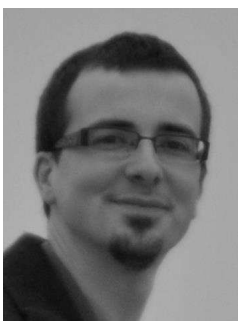

Jeroen Wyffels M.Sc. Jeroen Wyffels obtained his Master's Degree in Industrial Engineering Electronics - ICT at KAHO Sint-Lieven, Ghent, Belgium, in 2008. After his studies, he joined the DraMCo (wireless and mobile communications) research group. In 2010, he started his Ph.D. in the field of indoor localization in a healthcare setting, at the KU Leuven, Belgium, in close cooperation with Televic Healthcare, Izegem, Belgium.

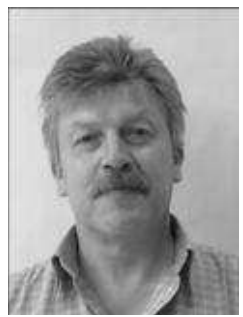

Jos De Brabanter Prof. Dr. Ing. Jos De Brabanter received the degree in Electronical Engineering (1990), Safety Engineer (1992), Master of Environment, Human Ecology (1993), Master in Artificial Intelligence in (1996), Master of Statistics (1997) and the Ph.D. degree in Applied Sciences (2004). His research interests are Non-parametric Statistics and Kernel Methods, areas in which he has several research papers. He is also co-author of the book "Least Squares Support Vector Machines" (World Scientific, 2002). Since 2008 he is an Associated Professor with the KU Leuven, Belgium.

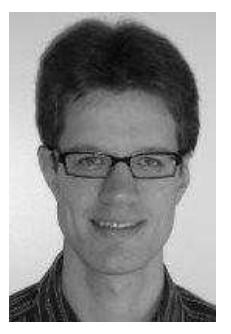

Pieter Crombez Dr. Ir. Pieter Crombez is a Senior R\&D engineer in Televics healthcare communication division where he is responsible for the wireless research for next generation nurse call products. Currently he is focusing on localization techniques that meet the harsh specifications for the healthcare market. Previously he worked as a Ph.D. researcher at the wireless group at IMEC, Leuven, Belgium on low power, reconfigurable transceivers for multi standard/multi mode applications. He also worked as a research assistant at the ESAT-MICAS laboratory of the KU Leuven University. Pieter Crombez is a valid member of IEEE and (co)author of several publications.

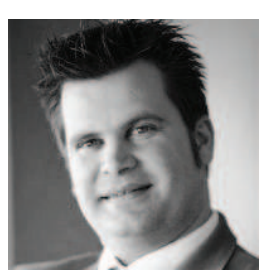

Piet Verhoeve Prof. dr. ir. Piet Verhoeve obtained a MSc. in Electrical Mechanical Engineering at KU Leuven (1991) and a Ph.D. in Electronic Engineering at Ghent University (1998), Belgium. He started as a software architect at Lernout \& Hauspie (a speech technology) before he joined Televic in 2001 , to set up the innovation process \& research collaboration. As of 2004 he explored the home care market and eHealth technologies(such as localization, reasoning, context awareness). In 2007 he became operational business lead for the Healthcare business unit. In 2011 he was appointed Director of Innovation\&Technology at Televic Group. In April 2013 he joined the iMinds research institute to lead he interdisciplinary research collaboration program (ICON). Piet Verhoeve is guest professor at the Multimedia Lab research - Ghent University and (co)author of several publications and patent applications. He is Sr. member of IEEE.

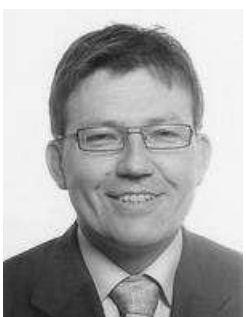

Bart Nauwelaers Prof. Dr. Ir. Bart Nauwelaers received the M.S. and Ph. D. degrees in electrical engineering from the KU Leuven, Belgium in 1981 and 1988, respectively. He also holds a Master degree in design of telecommunication systems from ParisTech Tlcom, France. Since 1981 he has been with the Department of Electrical Engineering (ESAT) of KU Leuven, where he has been involved in research on microwave antennas, passive components, interconnects, microwave integrated circuits and MMICs, linear and non-linear device modelling, MEMS and wireless communications. He is former chair of IEEE AP/MTTBenelux and past chair of URSI-Benelux. Bart Nauwelaers teaches courses on microwave engineering, on analogue and digital communications, on wireless communications and on design in electronics and telecommunications. For the last twenty years he has served education in several functions, the last one being programme director for the bachelor and master programmes in electrical engineering.

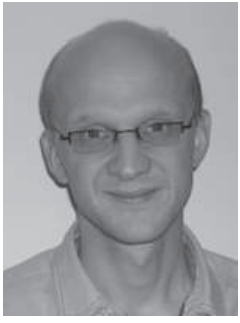

Lieven De Strycker Prof. Dr. Ir. Lieven De Strycker received the Master degree in Electrotechnical Engineering from Ghent University, Belgium, in 1996 and the Ph.D. degree in Electrotechnical Engineering from Ghent University, Belgium, in 2001. Since 2001 he is Professor with the Catholic University College Ghent, Belgium, where he founded, with his colleagues, the DraMCo (wireless and mobile communications) research group. Since 2008 he is also an associated Professor with the KU Leuven, Belgium. 